Impacto das Políticas Públicas na Promoção da Educação Sexual: o Caso Português Impact of Public Policy for the Promotion of Sex Education: The Portuguese Case

\author{
Ana Cristina Rocha \\ Cidália Duarte \\ Universidade do Porto
}

Palavras-chave: educação sexual, políticas, Portugal, modelo ecológico

Keywords: sexuality education, policies, Portugal, ecological model

Autor Biografias: Ana Cristina Rocha é investigadora no Centro de Psicologia da Universidade do Porto. É mestre e doutorada em Psicologia pela mesma universidade. Os seus interesses de investigação focam-se na educação sexual e no desenvolvimento psicossexual. Tem também estudado o desenvolvimento profissional dos psicólogos. É ainda psicóloga no Serviço de Consulta de Psicologia da Faculdade de Psicologia e de Ciências da Educação da Universidade do Porto. Cidália Duarte é Professora Auxiliar na Faculdade de Psicologia e Ciências da Educação da Universidade do Porto e investigadora no Centro de Psicologia da mesma Universidade. Os seus principais interesses de investigação centram-se na educação sexual e sexualidade ao longo da vida, nas relações de intimidade amorosa e psicoterapia de casal. É igualmente a Coordenadora Cientifica do Serviço de Psicoterapia da FPCE.

Nota de Autor: Ana Cristina Rocha e Cidália Duarte, Faculdade de Psicologia e de Ciências da Educação e Centro de Psicologia, Universidade do Porto, Portugal. Este trabalho foi financiado pela Fundação para a Ciência e Tecnologia, Ministério da Educação e Ciência [SFRH/BD/74347/2010].

Citar: Rocha, A.C., and Duarte, C. (2016). Impacto das Políticas Públicas na Promoção da Educação Sexual: o Caso Português. Global Journal of Community Psychology Practice, 7(1S), 1-22. Retrieved Day/Month/Year, from (http://www.gjcpp.org) 


\section{Impacto das Políticas Públicas na Promoção da Educação Sexual: o Caso Português}

\section{Resumo}

A Europa é, provavelmente, o continente com a mais longa experiência no domínio da educação sexual. Contudo, são as experiências desenvolvidas em território norteamericano que dominam os estudos de avaliação do impacto da educação sexual. Segundo o gabinete europeu da OMS, esta dominância deve-se, sobretudo, à ausência, na Europa, de um processo de publicação sistemática das experiências e resultados nacionais no contexto internacional.

É neste âmbito que nos propomos a partilhar o caso de Portugal relativamente às políticas de promoção da educação sexual, com base numa perspetiva (bio)ecológica. Portugal tem um percurso longo e algo errático neste domínio, tendo-se verificado, nas últimas três décadas, no plano crono- e macrossistémico, notáveis mudanças ao nível das medidas políticas e legislativas, que culminaram na conceção da mesma como um projeto comunitário. No plano do exo-, meso- e microssistema, estas mudanças refletem-se nas práticas.

A percentagem de escolas portuguesas a implementar a educação sexual tem vindo a aumentar e, não obstante alguma variabilidade, há práticas em comum nas escolas, sobretudo no que respeita os papéis/funções desempenhados e os procedimentos de planeamento, implementação e avaliação. Há também dificuldades comuns como: envolver a comunidade escolar, implementar a transversalidade, abordar a sexualidade de forma holística e avaliar adequadamente a educação sexual. Estas dificuldades são potenciadas também por medidas politico-legislativas no domínio da educação, como corroboram as perceções da comunidade escolar e científica. Ambas receiam, atualmente, que a sustentabilidade da educação sexual nas escolas enquanto projeto comunitário esteja em causa, devido às últimas políticas educativas. Neste sentido, e com base em referências teóricas da intervenção comunitária, finalizamos este artigo apresentando uma reflexão sobre os desafios atuais no domínio, bem como um conjunto de propostas para ultrapassar os mesmos. 


\title{
Impact of Public Policy for the Promotion of Sex Education: The Portuguese Case
}

\begin{abstract}
Europe has probably the greatest experience in sexuality education. However, in the most recent research on its impact evaluation, the majority of studies focused on experiences in US territory. According to the European Office of WHO, this dominance happens due to the absence in Europe of a systematic publishing process of national experiences and results on the international context.

Therefore, we propose to share the case of Portuguese policies in promoting sexuality education, based on a (bio) ecological approach. Implementation of sexuality education in Portugal has been long and somewhat erratic. At chrono- and macrosystem levels, the last three decades have been marked by changes in policies and laws, and sexuality education has been view as a community project. These changes impact on practices, at meso and microsystem levels.

The percentage of Portuguese schools implementing sexuality education has been increasing and, despite some variation, there are common practices in schools, especially those related to roles/functions played by stakeholders, and planning, implementation and evaluation procedures. There are also common difficulties such as: low school community participation, cross-curricular teaching, too heavy a focus on health-related issues, and poor-quality evaluation. These difficulties are also maximized by the educational policies and legislation, and validate the perceptions of academic and scientific community. Both fear that today the sustainability of school-based sexuality education as a community project is endangered because of the latest educational policies. So, in the end of this paper, we present an analysis of the current challenges in sexuality education, based on theoretical references of community intervention, as well as a set of proposals to overcome them.
\end{abstract}




\section{Introdução}

Ainda que em diferentes momentos, a educação sexual nasceu, em todo o mundo, por necessidades sociais despoletadas ora pela revolução sexual e pela emergência da adolescência, na Europa ocidental dos anos 60/70; ora por prevenção epidémica do VIH, nos anos 80/90, nos Estados Unidos da América e, mais tarde, na América Latina, Ásia e África. As questões sociais, que marcaram o seu início, influenciaram também todo o seu desenvolvimento, acentuando diferenças entre continentes ainda na atualidade (Ketting \& Winkelmann, 2013; WHO/BZgA, 2010).

Na Europa, após séculos de Cristianismo, a sociedade, com a influência dos trabalhos desenvolvidos na sexologia (ex., Havelock Ellis, Freud e Kinsey), tornou-se menos repressiva (Loeber, Reuter, Apter, van der Doefx, Lazdane, \& Pinter, 2010; WHO/BZgA, 2010), evidenciando-se a necessidade da educação sexual formal. Esta, tendo-se iniciado na Suécia, em 1955, propagou-se por vários países do ocidente nas décadas de 70 e 80 e surgiu, no centro e leste europeu, apenas depois da queda do comunismo. Ao longo das últimas décadas, por oposição ao que acontece nos Estados Unidos da América, tem havido, na Europa, consenso científico relativamente à abordagem holística, baseada nos direitos sexuais e numa conceção abrangente e positiva da saúde sexual, por oposição ao modelo moral e de risco implícitos na abordagem centrada na abstinência que predomina nos Estados Unidos da América (Ketting \& Winkelmann, 2013; Sanchéz, 2005).

Contudo, a avaliação do impacto da educação sexual, com destaque para as meta-análises de Kirby (2008) e da UNESCO (2009), é dominada por estudos em território norte-americano; o que, segundo o gabinete europeu da OMS (WHO/BZgA, 2010), evidencia a lacuna na documentação e partilha do trabalho desenvolvido na Europa. Perante esta lacuna, foi criado precisamente o gabinete regional supracitado, com o objetivo de delinear uma estratégia comum para a saúde sexual e reprodutiva. Não obstante os esforços de homogeneização da educação sexual, nomeadamente através da publicação de guidelines para a sua implementação (WHO/BZgA, 2010; 2013), a informação mais recente sobre o estado da educação sexual na Europa foi publicada num guia de referência há aproximadamente 10 anos. Ainda que este não se baseie em dados recolhidos no terreno sobre as práticas na educação sexual, demonstrou tanto a variabilidade entre países como a existência de pontos em comum. Especificamente, este pôs em relevo países como França, Bélgica e Portugal, que têm um longo percurso politico-legislativo no domínio e que, depois de terem tornado a educação sexual obrigatória nas escolas, reúnem esforços para a implementar efetivamente e ultrapassar dificuldades, algumas das quais também comuns a diversos países. Portugal constitui-se, assim, um caso relevante para a análise do impacto das políticas públicas na promoção da educação sexual.

\section{Implementação da Educação Sexual no Contexto Escolar Português}

Como referimos, o caso português inserese no contexto europeu, no qual, não obstante as diferenças politicolegislativas e práticas, a educação sexual tem sido perspetivada, por várias organizações e instituições, como uma responsabilidade de toda a comunidade escolar, apoiada por uma rede de 
parceiros, e não como uma iniciativa individual, nomeadamente de um professor ou de uma Organização NãoGovernamental [ONG] (IPPF, 2010; UNESCO, 2010; WHO/BZgA, 2010; 2013). É assim, colocando a tónica na comunidade e na rede de apoio, que a educação sexual deixa de ter como únicos alvos de mudança desenvolvimental os sistemas pessoais (do estudante) e passa a ser um projeto da comunidade e de intervenção comunitária no contexto escolar (Campos, 1991). Esta evolução no sentido da superação do viés individualista e intrapsíquico na Psicologia, permeio do reconhecimento da necessidade de se intervir intencionalmente em dimensões contextuais, como advoga a Psicologia Comunitária (Orford, 1992), foi acompanhada no domínio da promoção da saúde. Iniciou-se na Carta de Ottava (OMS, 1986), na qual se preconizou uma abordagem sociológica da saúde, e progressivamente se estendeu à educação para a saúde (McLeroy, Bibeau, Steckler, \& Glanz, 1988) e, ainda que menos frequentemente, à educação sexual, (Eisenberg, Madsen, Oliphant, \& Resnick 2012).Os princípios da rede europeia de escolas promotoras de saúde, que Portugal integrou, refletem precisamente a adoção desta abordagem (European Network of Health Promoting Schools, WHO, \& European Commission, 1997).

Pelo contributo incontornável que deu para o estudo do desenvolvimento em contexto e, consequentemente, para a intervenção (psicológica) dirigida ao contexto (comunitário),a abordagem (bio)ecológica, desenvolvida por Bronfenbrenner $(1979,2005)$, é bastante pertinente para a análise da educação sexual nas escolas. Pois, esta abordagem não só permite sustentar a relevância e pertinência da educação sexual como um projeto comunitário, como permite também clarificar a natureza distinta, ainda que interrelacionada, entre o processo da sua implementação em contexto escolar e o processo inerente à própria educação sexual, abarcando a complexidade de ambos decorrerem em contexto. De acordo com a mesma, a educação sexual será um processo proximal estimulante do desenvolvimento psicossexual a decorrer no microssistema escolar ao longo do tempo. Assim, enquanto interação recíproca entre, neste caso, principalmente estudante e meio ambiente, este processo proximal é influenciado por ambos. Portanto, não é apenas o próprio desenvolvimento psicossexual do estudante que é influenciado pelo contexto imediato da escola e pelas influências e interconexões entre contextos/sistemas mais vastos, mas também a própria implementação da educação sexual em contexto escolar enquanto processo proximal.

A implementação da educação sexual enquanto processo proximal, no que diz respeito ao contexto, é então influenciada: 1) ao nível do cronossistema, pela evolução histórica e pelas experiências prévias referentes à educação sexual e conceitos contíguos, especialmente em Portugal e na Europa; 2) ao nível macrossistémico, pelo repertório cultural e sistema de crenças vigente dos quais decorrem, por exemplo, as medidas políticas e a legislação; 3) ao nível do exossistema, pelas relações e processos que ocorrem entre as escolas e outras instituições (ex. ONG, estruturas intermédias do Ministério da Educação e Ciência [MEC]); 4) ao nível do mesossistema, pelas mesmas relações e 
processos, mas entre as escolas e contextos que incluem o estudante (ex. família); e 5) ao nível do microssistema pelas estruturas e processos na escola que, por sua vez, é um sistema com vários níveis interrelacionados e compostos por vários processos de regulação (ex. organizacional, interpessoal, intrapessoal) (Bronfenbrenner, 1988; Eccles \& Roeser, 2010).

\section{Evolução das políticas públicas: crono- e macrossistema}

0 percurso da implementação da educação sexual nas escolas, em Portugal, é longo, tem sido percecionado como errático, descontínuo e inconcluso (Baptista, 2014), e está repleto de exemplos da influência de fatores alocados nos diversos sistemas ecológicos. Entre o século XIX e a revolução que pôs fim à ditadura, em 1974, na sociedade portuguesa, prevaleceu, resultado da forte influência da igreja católica, uma ideologia fortemente repressiva e fóbica relativamente à sexualidade que enfatizava valores como o da castidade (Sousa, 2012). Todavia, na década de 60, a influência da revolução sexual começou a fazer-se sentir, tendo-se fundado a Associação para o Planeamento da Família [APF]. Posteriormente, com o fim da ditadura, teve início uma progressiva reforma moral nas várias camadas da sociedade (Vaz, Vilar, \& Cardoso, 1996).

Como em muitos dos países do ocidente europeu, as primeiras medidas políticas para promover a educação sexual ocorreram na década de 70 através da criação da 'Comissão sobre Educação e Sexualidade', que foi rapidamente extinta, mas também da nova Constituição da República Portuguesa, que criou a base jurídica para o início da promoção e educação para a saúde sexual no país. Contudo, é da década de 80 que data o primeiro grande marco legislativo neste domínio: a Lei no 3/84 e a Portaria no 52/85. Estas determinaram, respetivamente, a garantia do direito à educação sexual como componente do direito fundamental à educação, através do estabelecimento de programas escolares, e o regulamento das consultas de planeamento familiar e dos centros de atendimento para jovens (Sousa, 2012; Vaz et al., 1996; Vilaça, 2013).

Contudo, esta lei não acarretou grande avanço e a educação sexual apenas passou a estar no centro da discussão novamente aquando do debate da Lei de Bases do Sistema Educativo em 1986 (Lei no 46/86), que, aliando os saberes académicos ao desenvolvimento pessoal e social, estabeleceu nos currículos escolares uma nova área de formação pessoal e social, na qual a educação sexual poderia ser incluída. Esta nova área foi, porém, apresentada como alternativa à educação religiosa. Já, nos anos 90, surgiram propostas para o programa da área de desenvolvimento pessoal e social, bem como diversas iniciativas por parte do Ministério da Educação, entre as quais o 'Programa de Promoção e Educação para a Saúde' [PPES] e a adesão à 'Rede Europeia de Escolas Promotoras de Saúde'. Houve também, nesta fase, um esforço por uma articulação entre o Ministério da Educação e o da Saúde (Despacho Conjunto no 271/98) (Sousa, 2012; Vaz et al., 1996; Vilaça, 2013).

Em 1995, a APF propôs uma parceria com o PPES que visava a realização de um estudo experimental em cinco escolaspiloto. Não obstante nunca ter sido generalizado a outras escolas, devido a pressões das alas mais conservadoras, no 
âmbito deste estudo foram elaboradas as orientações técnicas (Marques, Pereira, Silva, Vilar, \& Cadete, 1999), que vieram, posteriormente, a servir de base para as "Linhas Orientadoras da Educação Sexual em Meio Escolar", assinadas pelos Ministérios da Educação e da Saúde e pela APF, em 2000, no âmbito da publicação de nova legislação no domínio: Lei $\mathrm{n}^{\mathrm{o}}$ 120/99 e Decreto-Lei no 259/2000.

Esta legislação procurou garantir o desenvolvimento da educação sexual através da responsabilização das escolas pela sua integração no projeto educativo, que deveria ser concretizado nos projetos de turma, em colaboração com os serviços locais de saúde e as associações de estudantes e as de pais/encarregados de educação, i.e. atribuiu à educação sexual uma dimensão comunitária, assumindo que a mesma se desenvolve em contexto. Reforçou também a abordagem dos conteúdos interdisciplinar e adequada à faixa etária dos estudantes, bem como a importância da formação de professores. Ainda no final dos anos 90, o Ministério da Educação formou a Comissão de Coordenação da Promoção e Educação para a Saúde, que envolvia as Direções Regionais de Educação [DRE], na articulação com o terreno (Baptista \& Matos, 2011a; Vilaça, 2013), reconhecendo, assim, a influência de sistemas mais distantes da escola.

No início dos anos 2000, facilitou-se a implementação das 'Linhas Orientadoras' através da criação das áreas curriculares não disciplinares (Decreto-Lei no 6/2001) e do estabelecimento de protocolos com $\mathrm{ONG}$, entre as quais a APF, que tinham como intuito o apoio por parte destas ao desenvolvimento da educação sexual em meio escolar. Conquanto, a este período seguiu-se um desinvestimento na área entre 2003 e 2005, que findou com a criação de um grupo de especialistas, denominado por Grupo de Trabalho em Educação Sexual [GTES]. Este foi justificado pelas dificuldades da aplicação da Lei no 120/1999 e documentos relacionados, assim como pela sensibilidade com que a temática era recebida na opinião pública, aludindo à polémica despoletada pelos mass media.

Este grupo, cuja missão era "estudar e propor os parâmetros gerais dos programas de educação sexual em meio escolar, na perspectiva da promoção da saúde escolar" (Despacho nำ19 737/2005), publicou dois relatórios principais, em 2005 e 2007, nos quais, de forma complementar, fez uma descrição das práticas no âmbito da educação para a saúde/sexual e da literatura científica relevante para a sua implementação em meio escolar. Nos mesmos, sugeriu um conjunto de medidas a adotar com impacto desde o nível microssistémico escola ao nível macrossistémico Ministério da Educação. As recomendações do GTES estiveram na base da mais recente legislação portuguesa sobre educação sexual: a Lei no 60/2009 e a respetiva Portaria no 196A/2010 (Baptista \& Matos, 2011b; Vilaça, 2013).

Assim, segundo esta legislação, a educação sexual é uma componente da educação para a saúde a ser integrada obrigatoriamente no projeto educativo da escola ou agrupamento de escolas. As finalidades, os conteúdos mínimos obrigatórios ao longo de todos os ciclos e a carga horária mínima da educação sexual são também estabelecidos pela Lei no 60/2009 e devem ser cumpridos nas áreas curriculares não disciplinares e também transversalmente nas disciplinas. 
Esta legislação estabelece ainda as funções do gabinete de informação e apoio.

A nível microssistémico, a lei indica que um professor deve ser nomeado para coordenar uma equipa interdisciplinar, que é responsável por dinamizar a educação para a saúde/sexual. Este professor-coordenador, cuja formação específica foi garantida pelo Ministério da Educação, usufruiria da redução da componente letiva, sendo a sua seleção baseada em critérios como a formação específica e a experiência no domínio da educação sexual. Já o projeto de educação sexual de cada turma deve ser desenvolvido pelo conselho da respetiva turma, com destaque para os professores e o diretor da mesma. Sem prejuízo do anterior, a legislação prevê o estabelecimento de parcerias e um papel ativo para a comunidade escolar, particularmente para as associações de pais/encarregados de educação e estudantes, reforçando assim o caráter comunitário do projeto.

A nível mesossistémico, segundo esta lei, os encarregados de educação devem ser, no mínimo, informados de todas as atividades no domínio; e a nível exossistémico, as escolas devem ter o acompanhamento das unidades locais de saúde e podem ter $\mathrm{ONG}$, reconhecidas e especializadas na área, como parceiras. Por fim, a nível macrossistémico, o Ministério da Educação é responsável por supervisionar, coordenar e avaliar a educação para a saúde/sexual nas escolas, bem como por elaborar relatórios de avaliação periódica com base, nomeadamente, em questionários realizados nas escolas. Um relatório global sobre a aplicação desta legislação deveria ter sido realizado em 2011, dois anos letivos após a publicação da Lei no $60 / 2009$. 0 mesmo só foi publicado no final de 2013 (Matos, Reis, Ramiro, Ribeiro, Leal, \& Equipa Aventura Social, 2013).

Desde a publicação da Lei no 60/2009 e respetiva Portaria, nenhuma medida política específica da educação sexual foi adotada. Todavia, o mesmo não se sucedeu no contexto global da educação. As medidas no âmbito da revisão da estrutura curricular e da lei da autonomia acarretaram implicações diretas e consideráveis para a educação sexual. A chamada 'instrução' foi valorizada na revisão da estrutura curricular em detrimento da cidadania e do desenvolvimento pessoal e social do estudante. Pois, o Despacho no 17169/2011, que finda a aplicação do documento 'Currículo Nacional do Ensino Básico - Competências essenciais', terminou com a obrigatoriedade das áreas curriculares não disciplinares. A cidadania passou, assim, para o plano da intenção educativa, dependente do tempo/espaço que os agrupamentos de escolas lhe pretendam e possam conferir; já que o Decreto-lei no 139/2012 estabelece que "as escolas, no âmbito da sua autonomia, devem desenvolver projetos e atividades que contribuam para a formação pessoal e social dos alunos, designadamente a educação para a saúde".

Conforme o estabelecido no Despacho normativo 13-A/2012 sobre os mecanismos do exercício da autonomia pedagógica e organizativa, cada escola pode gerir os tempos letivos, a carga curricular de cada disciplina, assim como as ofertas curriculares obrigatórias ou complementares, mediante o crédito de tempos que lhe é atribuído. Todavia, estes 
créditos, por sua vez, dependem, entre outros, da progressão dos resultados escolares, da aferição dos resultados internos com os externos e do sucesso escolar alcançado pelos alunos. Portanto, a autonomia que as escolas possuem é, na verdade, reduzida devido ao requisito de obterem determinados resultados que não dependem unicamente das ações sob o seu controlo (ex. sucesso escolar). Há, então, uma negação da relação intrínseca entre saúde e educação, defendida nomeadamente pela OMS e pela União Internacional de Promoção da Saúde e de Educação para a Saúde [UIPES] (2010), e uma negação da influência ecológica de outros sistemas que não o escolar no sucesso escolar. Há também uma gestão centralizada e autocrática que não envolve efetivamente os profissionais do contexto na tomada de decisões, sendo que, como apontam Foster-Fishman e Keys (1997), os contextos geridos desta forma tendem a resistir à mudança apesar das iniciativas transformadoras, como, neste caso, pretende ser a legislação da educação sexual enquanto projeto comunitário.

Naturalmente que a importância dos saberes académicos é amplamente reconhecida, mas não como um fim encerrado em si mesmo. Estes devem ser integrados numa educação holística, que capacita os jovens com conhecimentos e competências que lhes permitam exercer a sua cidadania. Neste seguimento, a atual lei da autonomia é implicitamente perversa, pois, se num primeiro momento assegura a autonomia das escolas para a definição das necessidades do seu contexto específico, num segundo momento impõe autocraticamente determinadas necessidades associadas aos saberes puramente académicos como prioritárias. Assim, como salienta Baptista (2014), esta legislação pode acentuar assimetrias entre escolas e comunidades, pois aquelas com resultados académicos satisfatórios poderão promover o desenvolvimento pessoal e social da sua comunidade e aquelas com resultados académicos insatisfatórios não o poderão fazer, contribuindo potencialmente para a pioria dos próprios resultados académicos. Por conseguinte, o percurso evolutivo das políticas de educação sexual em Portugal parece continuar a ser marcado por descontinuidades, como refere a autora supracitada, e ilustra a influência do crono- e macrossistema na implementação da educação sexual nas escolas.

\section{Impacto nas práticas: exo-, meso- e microssistema escolar}

Não obstante o aumento considerável do número de estudos dedicados à temática nos últimos anos, a informação sobre a implementação da mesma no contexto escolar português é ainda parca. Ainda assim, a investigação tem sido unânime quanto à evidência dos efeitos positivos da educação sexual, sobretudo no que respeita as práticas de risco (Ramiro, 2013; Ramiro et al., 2011; 2014; Reis, 2012; Reis et al., 2011; 2013; Sousa et al., 2007). Concomitantemente, os estudos mais recentes focados nas práticas têm evidenciado procedimentos e fatores em comum, mas também variabilidade entre escolas (ex. Alves, 2012; Dias, 2013; GTES, 2005; 2007; Leal, 2013; Macário, 2010; Matos et al., 2011; 2013; Ramiro, Reis, Matos, \& Diniz, 2013, Rocha \& Duarte, 2015a,b; Rocha, Leal, \& Duarte, 2015).

A esmagadora maioria das escolas/agrupamentos leva a cabo a 
implementação da educação sexual através da sua integração no projeto educativo no âmbito da educação para a saúde. A nível microssistémico, a sua implementação tem vindo a aumentar ligeiramente ao longo dos anos (Abrantes et al., 2013; GTES. 2005; 2007; Matos et al., 2011; 2013; Ministério da Educação, 2009; 2010; 2011); aliás um terço das escolas iniciou-a aquando da publicação da última legislação, o que evidencia a influência considerável dos fatores macrossistémicos (Rocha \& Duarte, 2015a). Cerca de 83\% das escolas/agrupamentos cumpre a carga horária mínima estipulada por lei (Matos et al., 2013). Contudo, mais de metade destas não dispõe de orçamento para a educação sexual/saúde (Matos et al., 2011; Rocha \& Duarte, 2015a), que, não sendo crucial, é um fator facilitador da mesma (Rocha \& Duarte, 2015b). De forma global, as direções nomeiam um professor coordenador, maioritariamente com formação na área das ciências experimentais, que coordena uma equipa, por vezes, multidisciplinar, mas que em apenas $42 \%$ das escolas integra o psicólogo (Matos et al., 2013; Ramiro et al., 2013; Rocha \& Duarte, 2015a, Rocha et al., 2015).

0 planeamento é da responsabilidade primordial desta equipa e desenrola-se de forma a que a educação sexual seja adequada ao contexto específico de implementação, ainda que este procedimento seja pouco sistemático. Alguns estudos têm demonstrado precisamente que a participação da comunidade é baixa e que os procedimentos para a sua promoção são muito pouco regulares (ex. avaliação das necessidades e interesses) (Alves, 2012; Matos et al., 2013; Rocha \& Duarte, 2015a; Rocha, et al., 2015). Assim, os objetivos e os conteúdos da educação sexual são estabelecidos sobretudo pela equipa de coordenação, que também é a principal responsável por selecionar a metodologia e as estratégias de implementação a serem posteriormente adaptadas a cada turma pelos professores.

Após a reforma curricular, especificamente o fim da obrigatoriedade das áreas curriculares não disciplinares, que eram, até $2011 / 12$, o principal espaço de alocação dos conteúdos da educação sexual, a metodologia mais implementada passou a ser a transversalidade, sendo a educação sexual em cada turma dinamizada principalmente pelos professores (Rocha \& Duarte, 2015a; Rocha et al., 2015). Todavia, vários estudos têm apontado para a dificuldade em conseguir-se uma total integração dos conteúdos da educação sexual nos curricula das diversas disciplinas, já que a transversalidade, por vezes, se aplica apenas às áreas disciplinares de Ciências Naturais, Educação Física e Educação Moral e Religião Católica (Alves, 2012; Dias, 2013; Macário, 2010; Matos et al, 2011; 2013; Rocha \& Duarte, 2015a; Rocha et al., 2015). Não obstante as estratégias pedagógicas planeadas para o contexto de sala de aula atribuírem aos estudantes maioritariamente um papel ativo, as atividades extracurriculares, sobretudo as palestras e conferências, são também bastante utilizadas. Estas constituem, quase exclusivamente, os únicos momentos de promoção da participação da comunidade, uma vez que a formação de agentes é também pouco regular (Matos et al., 2011; 2013; Rocha \& Duarte, 2015a; Rocha et al., 2015). 
Adicionalmente, os estudos têm consistentemente assinalado um enviesamento, nos conteúdos abordados, no sentido da componente biológica e de uma perspetiva negativa e informativa da sexualidade, ainda que a natureza holística da sexualidade não seja totalmente negligenciada (Alves, 2012; Dias, 2013; Macário, 2010; Rocha \& Duarte, 2015a; Rocha et al., 2015). Portanto, no que diz respeito à implementação, as guidelines internacionais sobre o envolvimento da comunidade, a utilização de estratégias ativas, a abordagem abrangente e positiva da sexualidade e a alocação de espaço/tempo no curriculum escolar, não sendo completamente aplicadas, também não são totalmente descuradas.

Já a avaliação da educação sexual encontra-se ainda numa fase bastante incipiente. Não obstante ser planeada, esta centra-se quase exclusivamente nos professores e abarca apenas indicadores como o número de horas lecionadas, a percentagem de atividades realizadas e o nível de participação e satisfação quanto às atividades extracurriculares (Alves, 2012; Matos et al., 2013; Rocha \& Duarte, 2015a; Rocha et al., 2015); excluindo, portanto, a avaliação do impacto e da eficácia da mesma nos estudantes, bem como nos restantes elementos da comunidade.

No estudo sobre o impacto da legislação mais recente (Matos et al., 2013), a generalidade dos agentes escolares inquiridos considerou a adesão dos professores e dos alunos à educação sexual muito boa, a adesão da comunidade escolar em geral satisfatória e a dos pais, respetiva associação e associação de estudantes oscilou entre satisfatória e fraca. Resultados de outros estudos (Alves, 2012; Rocha \& Duarte, 2015a; Rocha et al., 2015) corroboram a evidência de que não há o envolvimento de todos os agentes educativos, sendo que o dos alunos aumenta sobretudo aquando da fase de implementação. Na verdade, apesar de cerca de metade dos diretores dos agrupamentos considerarem que a educação sexual tem sido progressivamente integrada na cultura das escolas, estes assinalam o envolvimento da comunidade como uma dificuldade, devida ao facto de não fazerem parte da cultura dominante nem a comunicação escola-pais, nem a comunicação e colaboração entre direções, professores e alunos. A cooperação das direções, a participação dos alunos no planeamento, a formação frequente de professores e o estabelecimento de parcerias com entidades externas foram as estratégias mais apontadas por estes para promover o envolvimento da comunidade (Matos et al., 2013).

Todavia, ao nível do exossistema, apesar de ser uma prática bastante comum em 90\% das escolas (Dias, 2013; Matos et al., 2011; 2013; Rocha \& Duarte, 2015a; Rocha et al., 2015), o estabelecimento de parcerias tanto tem sido reconhecido como um facilitador da implementação efetiva da educação sexual (Rocha \& Duarte, 2015b), como também tem sido apontado como um obstáculo à participação da comunidade, uma vez que alguns parceiros não promovem e apoiam o envolvimento da comunidade, mas substituem-na executando o projeto sem a participação ativa da mesma (Rocha, 2015; Rocha et al., 2015;). Ainda neste nível, as escolas sentem-se pouco apoiadas por parte das DRE, desconhecendo, mais de um terço, 
inclusivamente medidas de apoio por parte destas estruturas, o que justifica o baixo nível de satisfação relativamente às mesmas (Rocha \& Duarte, 2015a).

Concluindo, os diversos estudos têm demonstrado que a implementação da educação sexual tem vindo a aumentar nos últimos anos, mas, além da variabilidade entre escolas, algumas dificuldades e fragilidades parecem persistir, entre as quais: dificuldades em descentrar-se da componente biológica da sexualidade, em implementar o modelo transversal, em promover a participação da comunidade, sobretudo dos pais e associações representativas, e em efetuar uma avaliação adequada. A equipa responsável pelo relatório já mencionado referiu-se a um "panorama nacional de grande esforço pessoal e profissional por parte de direções e dos professores" para fazer cumprir a lei (Matos et al., 2013, p. $3)$ e, na sua análise global, apontando também forças, elenca várias fraquezas, algumas das quais corroboradas por outros autores (Alves, 2012; Dias, 2012; Macário, 2010; Rocha \& Duarte, 2015a; Rocha et al., 2015).

\section{Análise Global do Impacto das Políticas}

A publicação da última legislação, que é do conhecimento de $96 \%$ das escolas, tem sido reconhecida por estas como um importante facilitador da implementação da educação sexual (Matos et al., 2013; Rocha, 2015), ainda que as mesmas a considerem, numa escala de 5 pontos, apenas 3.72 clara e 2.95 exequível (Rocha \& Duarte, 2015a,b). Para além da considerável percentagem de escolas que referem ter começado a implementar a educação sexual aquando da sua publicação (Rocha \& Duarte, 2015a), não há forma de analisar, de modo rigoroso, o impacto da última legislação devido à escassez de dados científicos sobre a implementação da educação sexual nas escolas portuguesas. A evidência científica referente ao impacto das mudanças nas políticas educativas, em especial da lei da autonomia e da reforma curricular, é ainda menor, se não nula. $\mathrm{Na}$ verdade, reduz-se atualmente à diminuição do número de escolas que deixaram de abordar conteúdos da educação sexual nas áreas curriculares não disciplinares (Rocha \& Duarte, 2015a; Rocha et al., 2015). Ainda assim, quer os resultados da escassa investigação, quer a comunidade escolar têm evidenciado um impacto positivo, especialmente da legislação específica, mas também um negativo, em particular das políticas educativas posteriores (Baptista, 2014; Matos et al., 2013; Ramiro et al., 2013; Rocha \& Duarte, 2015a,b; Rocha et al., 2015).

Com efeito, o macrossistema parece contribuir também para as principais dificuldades identificadas na sua implementação. Pois, nos documentos oficiais nem constam os possíveis procedimentos para o envolvimento da comunidade, ainda que a responsabilidade da educação sexual lhe seja atribuída e a importância da sua participação seja salientada, nem o papel de cada agente é esclarecido, o que também se reflete posteriormente no estabelecimento de procedimentos a nível microssistémico, e no desempenho de funções por parte de parcerias e estruturas intermédias do MEC, ao nível do exossistema (Rocha \& Duarte, 2015a,b; Rocha et al., 2015).

Efetivamente, um dos princípios básicos da intervenção comunitária é o de que o processo de planeamento se trata de uma análise do contexto realizada juntamente 
com a comunidade e que culmina com a tomada de decisões num ambiente participativo, implicando, por isso, quer tempo para o cumprimento das diferentes fases inerentes, quer recursos humanos que funcionem como "ponte e não como trincheira" na promoção do envolvimento dos diferentes agentes educativos e, consequentemente, do desenvolvimento da comunidade (Becker, Edmundo, Nunes, Bonatto, \& Souza, 2004; Illback, Zins, Maher, \& Greenberg, 1990; Menezes, 2010; Nelson \& Prilleltensky, 2007).

Contudo, segundo os estudos supracitados, na realidade, deparamo-nos com o seguinte: 1) a avaliação das necessidades é um procedimento pouco sistemático e, quando realizado, é levado a cabo apenas por um agente (equipa/professores da turma) junto dos alunos; 2) o psicólogo, um dos profissionais com um perfil a priori mais adequado para trabalhar com a comunidade, potenciando o seu envolvimento e os seus recursos, não é integrado na maioria das equipas coordenadoras do projeto e, quando o é, divide o seu tempo com uma multiplicidade de tarefas desfavorável ao tempo exigido para o estabelecimento das relações basilares da sua intervenção; 3) o professor coordenador é selecionado com base na formação específica e não nas suas competências, nomeadamente no que respeita, por exemplo, o exercício de liderança e influência e o sentimento de controlo e autonomia, elementos centrais na promoção da mudança em contextos institucionais (Orford,1992); os parceiros perante as dificuldades da comunidade educativa em executar a legislação parecem substituir-se às comunidades, por oposição à constituição de uma parceria colaborativa e promotora do empoderamento da comunidade (Fawcett et al., 1995; Menezes, 2010; Zimmerman, 1995).

Por isso, e tal como reflete a apreciação dos diretores relativamente à ausência de comunicação (Matos et al., 2013), a comunidade não é envolvida "como agente ativo com voz, voto e veto", mas sim " como convidada, espetadora ou recetora de [supostos] benefícios" (Montero, 2004, p. 67). Naturalmente, não se identificando com o projeto, a comunidade participa pouco, como evidenciam os dados explanados anteriormente.

Da mesma forma, o macrossistema contribui para a centralização em determinados conteúdos, pois, ao contrário do recomendado pela comunidade científica (ex. Gómez-Zapiain et al., 2005; Sánchez, 2005), a legislação não define nenhum modelo teórico que sustente a intervenção, nem contradiz a abordagem sanitarista e redutora, o que a par do número reduzido e do caráter impreciso dos conteúdos mínimos obrigatórios descentrados da perspetiva biológica e negativa da sexualidade, pode contribuir para o foco excessivo nestes conteúdos. Concomitantemente, a transversalidade como única metodologia de integração da educação sexual nos curricula pode também contribuir para esta focalização excessiva na perspetiva biológica da sexualidade, pela sua redução à abordagem na disciplina de Ciências Naturais; sobretudo após o cancelamento da obrigatoriedade das áreas curriculares não disciplinares e da redução da componente letiva do professor coordenador, dificultando um planeamento dos conteúdos realizado com a comunidade, por falta de tempo para levantar as necessidades e partilhar 
as decisões (Menezes, 2010; Nelson \& Prilleltensky, 2005).

Concomitantemente, a opção por não explicitar um modelo teórico e um quadro ético de referência é, por sua vez, uma medida que não contribui para a facilitação da aplicação do modelo transversal. Pois, por um lado, suscita nos professores a incerteza sobre o que lhes é solicitado que executem e o receio de optarem por práticas eventualmente incorretas e que sobretudo fundamentem queixas por parte dos encarregados de educação, receios ilustrados no estudo de Ramiro e colegas (2013), e, por outro lado, permite que os professores adotem precisamente práticas menos eficazes, que são caraterísticas de outras abordagens/modelos teóricos não congruentes com a abordagem holística advogada na Europa, designadamente do modelo de risco. A focalização na vertente informativa e na componente fisiológica e negativa da sexualidade que, por sua vez, pode ter implícitos fundamentos da moral conservadora, ilustra a influência do modelo de risco. 0 vazio na atual legislação no que diz respeito ao modelo teórico e ao quadro ético, que deveriam fundamentar a intervenção, contribui, portanto, para a não homogeneidade entre as escolas relativamente a aspetos que não se tratam das especificidades do contexto/comunidade de cada escola, mas sim de evidências científicas sobre o que potencia a eficácia da educação sexual e da intervenção comunitária.

Todavia, a aplicação da metodologia transversal é também dificultada por outros fatores. Apesar de ter vindo a ser recomendada quer em Portugal (GTES, 2005; 2007), quer na Europa (IPPF, 2006), a transversalidade tem também sido reconhecida como um modelo exigente (Parker, Wellings, \& Lazarus, 2009). Esta tem sido recomendada essencialmente pelas vantagens teóricas de naturalizar a abordagem da sexualidade, envolver intencionalmente todos os professores, inclusive os que creem não serem educadores sexuais, introduzir e alocar eficientemente a educação sexual no curriculum. Todavia, estas vantagens teóricas têm colidido com barreiras práticas na sua aplicação, como: o perfil desadequado de alguns professores, agravado pela falta de formação específica ou pela ineficiência desta, a pressão para o cumprimento dos programas das disciplinas (tidas como essenciais), a desvalorização da educação sexual/para a saúde na avaliação das escolas, a responsabilização de todos sem clarificação dos papéis/funções de cada um, mas sobretudo sem a disponibilização das condições no que respeita a alocação de recursos humanos e de tempo/espaço para que a educação sexual seja desenvolvida, em rede, como um projeto da comunidade (fatores em grande parte da responsabilidade do MEC e fora do controlo das escolas). Estas dificuldades conduzem as escolas à realização de palestras por parte de parceiros para cumprimento das exigências legais (Matos et al., 2013), incapacitando a comunidade (Fawcett et al., 1995) e transformando a educação sexual numa área extracurricular, o que, segundo os resultados de um estudo de Rocha e Duarte (2015b) e a UNESCO (2010), é desfavorável a uma implementação mais efetiva da mesma.

Também a ausência de guidelines referentes aos procedimentos de avaliação da educação sexual, assim como o não cumprimento do legislado por parte do próprio MEC (ex. atraso na avaliação 
do impacto da última legislação), contribuem para a incipiência deste processo nas escolas, especificamente para que este se desenrole de uma forma desempoderante, uma vez que não assenta em princípios fundamentais como: a participação democrática, a melhoria e a pertença potenciada através da responsabilização da comunidade pelo mesmo (Becker et al., 2004; Illback et al., 1990; Menezes, 2010). Por fim, a insuficiência do apoio prestado pelas DRE contribui igualmente para a variância entre escolas, já que ele próprio é pouco homogéneo, como sugerido nos resultados do estudo de Leal (2013), que ilustraram diferenças estatisticamente significativas entre escolas pertencentes às DRE do Norte e de Lisboa e Vale do Tejo.

Por conseguinte, não obstante o impacto globalmente positivo do trabalho levado a cabo pelo GTES e da mais recente legislação no domínio da educação sexual, algumas das medidas consagradas na mesma (macrossistema) potenciam fragilidades e dificuldades vivenciadas ao longo dos restantes sistemas ecológicos. Estas dificuldades e fragilidades potenciadas pela legislação específica parecem ter sido agravadas consideravelmente pelo impacto negativo da escolha, por parte do MEC, pela 'dita instrução' como um fim encerrado em si próprio, na reforma curricular e nas posteriores mudanças no sistema educativo, colocando em causa a sustentabilidade da educação sexual nas escolas portuguesas como um projeto comunitário. A avaliação realizada pela comunidade escolar aponta neste sentido, já que a mesma refere que a Lei $n \underline{0}$ $60 / 2009$ e a respetiva portaria foram facilitadoras, mas muito desenquadradas das políticas educativas posteriores (Rocha, 2015).

De facto, no estudo qualitativo apresentado no relatório sobre o impacto da legislação (Matos et al., 2013), esta avaliação por parte da comunidade escolar é mais pormenorizada. Assim, as direções das escolas dizem-se sobrecarregadas com tarefas administrativas para cooperarem mais na educação sexual, embora reconheçam que, quando esta faz parte das rotinas e é sistematicamente acompanhada pelos diferentes órgãos, a resistência dos professores, pais e alunos diminui, sendo a sua implementação mais bem-sucedida, o que reforça o princípio básico da intervenção comunitária de que os profissionais e agentes da comunidade têm de ser envolvidos nas decisões para resistirem menos à mudança (FosterFishman \& Keys, 1997; Orford, 1992). Por seu turno, os professores sentem-se também sobrecarregados, pouco valorizados e reconhecidos, lamentando que o fim das áreas curriculares não disciplinares, assim como a não redução da componente letiva do professor coordenador, os tenha levado à rentabilização da disciplina das Ciências Naturais/Biologia para não comprometer o cumprimento dos programas das restantes disciplinas e ao estabelecimento de parcerias. Os alunos reconhecem os obstáculos a uma maior participação da sua parte causados pelo calendário eleitoral e pela instabilidade da sua associação representativa e, lamentando a repetição de temas, mostram-se pouco crentes da transversalidade devido ao perfil de alguns professores. Apresentam como propostas, nomeadamente, 0 recurso às novas tecnologias de informação e a participação de agentes 
externos. Os pais reconhecem os mesmos obstáculos relativos à sua associação, assim como as dificuldades inerentes a uma participação de pais que ultrapasse a resolução de problemas em exclusivo. $\mathrm{Na}$ verdade, os discursos dos diferentes agentes parecem refletir um baixo sentido de comunidade enquanto conceito que assenta nas componentes de pertença, influência, integração e satisfação de necessidades, e conexão partilhada (McMillan \& Chavis, 1986), o que pode concomitantemente ser causa e efeito da baixa participação da comunidade educativa em projetos como a educação sexual (Ramos, 2008).

Na verdade, a variância evidenciada em estudos recentes (Rocha \& Duarte, 2015a,b), a par das dificuldades em envolver a comunidade escolar, leva-nos a equacionar um eventual efeito perverso da Lei no 60/2009 na medida em que há a possibilidade de algumas escolas procurarem mais cumprir com os requisitos legais da obrigatoriedade da educação sexual, do que responder verdadeiramente às necessidades da comunidade escolar, como já equacionado por Sousa (2012); já que a educação sexual está concebida na legislação como um projeto da comunidade, mas as condições essenciais para uma intervenção comunitária, i.e., uma intervenção que promova o envolvimento ativo da própria comunidade (Menezes, 2010; Ramos, 2008), não são asseguradas na mesma. Neste sentido, também outros investigadores e os próprios dirigentes escolares receiam um retrocesso relativamente à sustentabilidade da educação sexual nas escolas (Baptista, 2014; Matos et al., 2013; Ramiro et al., 2013; Sousa, 2012).

\section{Desafios Atuais e Propostas de Ação Promotoras da Educação Sexual em Contexto Escolar}

0 panorama traçado anteriormente aponta para desafios na promoção da educação sexual no contexto escolar português, que não se restringem ao seu domínio específico, mas se propagam ao domínio lato da educação.

Essencialmente, identificamos na atualidade sete desafios interrelacionados e primordiais à educação sexual, nas escolas portuguesas.

0 primeiro não é específico da educação sexual, mas tem um impacto considerável na mesma e remete para a necessidade de valorizar o desenvolvimento pessoal e social dos alunos e das comunidades, reforçando a educação holística como forma de promover o empoderamento da comunidade educativa e envolvente (Menezes, 2010; Zimmerman, 1995).

0 segundo desafio atual resume-se à necessidade de assegurar as condições fundamentais para que a educação sexual se desenvolva efetivamente como um projeto comunitário, respondendo às necessidades específicas do contexto comunidade, escola, turma - no qual se desenvolve, e não aos indicadores legais inscritos na Lei no 60/2009 (Becker et al., 2004; Menezes, 2010); e potenciando a participação dos agentes da comunidade, nomeadamente dos estudantes e dos encarregados de educação, através de estratégias que vão ao encontro das suas necessidades e interesses e que fomentem a sua participação ativa, o que implica trabalhar com eles desde a conceção do projeto (Becker et al., 2004; Illback et al., 1990).

Bastante relacionado com os desafios anteriores, o terceiro desafio prende-se 
com a necessidade de potenciar o trabalho multidisciplinar através da complementaridade de saberes e competências dos diversos profissionais (Rappaport, 2005), sendo a este nível essencial adequar o número de recursos humanos alocados à equipa de coordenação, os períodos de trabalho da mesma, bem como os critérios de seleção destes recursos e a sua formação, que deve ser adequada especificamente ao papel desempenhado pelos diferentes profissionais na educação sexual.

Também como forma de promover o empoderamento da comunidade (Zimmerman, 1995), parece urgente aumentar a perceção de exequibilidade da legislação por parte da comunidade educativa - o quarto desafio. Esta pode ser potenciada através de medidas de apoio mais próximo e homogéneo por parte das estruturas intermédias do MEC, assim como da publicação de guidelines mais específicas que clarifiquem quer os procedimentos a adotar, especialmente no envolvimento da comunidade, na aplicação da transversalidade e na avaliação, quer o papel dos agentes educativos ao nível dos diversos sistemas ecológicos.

Providenciar condições estruturais que facilitem a integração da educação sexual no curriculum é o quinto desafio por nós identificado. Este pode ser respondido através, especificamente da:1) operacionalização e implementação do modelo transversal, e da interdisciplinaridade, entre as quais destacamos a valorização da educação sexual/para a saúde na avaliação das escolas (como advogam a OMS (1986) e a UIPES (2010)), a inclusão dos seus conteúdos nos programas das disciplinas, a formação específica dos professores, a atribuição de tempo para o planeamento e a monitorização; e 2) criação/reativação da obrigatoriedade de espaços curriculares inteiramente dedicados ao desenvolvimento pessoal e social do estudante, como era suposto serem as áreas curriculares não disciplinares.

Por fim, os dois últimos desafios referemse aos conteúdos e à avaliação.

Relativamente aos primeiros, será importante descentrá-los da vertente informativa e da componente biológica da sexualidade, sendo a definição do modelo teórico e do quadro ético um contributo essencial, conforme advogado por GómezZapiain e colaboradores (2005) e Sánchez (2005). No que concerne a avaliação, esta deve ser concetualizada como instrumento de empoderamento e, portanto de sustentabilidade da própria educação sexual e não de mero controlo, sendo essencial realizar-se a avaliação da eficácia e do impacto das atuais práticas como meio de análise do impacto das políticas atuais no domínio (Becker et al., 2004; Illback et al., 1990; Menezes, 2010).A este nível, e como também sugerido por Matos e colegas (2013), as entidades financiadoras de projetos de investigação e desenvolvimento, nomeadamente a tutelada pelo MEC, podem ter um papel fundamental no apoio a projetos de investigação, com especial destaque para projetos de investigação-ação que potenciam a relação universidade-escola na construção multidisciplinar do conhecimento relativo a intervenções empiricamente validadas (Oja \& Smulyan, 1989).

Assim, importa esclarecer que às propostas que associamos aos desafios elencados anteriormente, sobretudo às de definição de modelos e procedimentos, 
não subjaz nenhuma recomendação no sentido da regulamentação de códigos de conduta obsessivos que tolham a autonomia e o poder de decisão das comunidades escolares, instituições parceiras e estruturas intermédias do MEC. 0 que pretendemos são, antes, propostas de medidas políticas que potenciem precisamente quer a tomada de decisões por parte das comunidades escolares, tendo em vista a sua saúde sexual (WHO, 2006), quer o apoio às mesmas por parte de parceiros e estruturas intermédias do MEC. 


\section{Referências}

Abrantes, E., Ladeiras, L., Manso, M., \& Lima, R. M. (2013). Relatório 2012/2013: Promoção e educação para a saúde. Lisboa: DGE. Retirado de: http://www.dgidc.minedu.pt/educacaosaude/index.php?s $=$ directorio\&pid $=188$

Alves, G. (2012). Modelos em educação sexual e práticas de professores dos

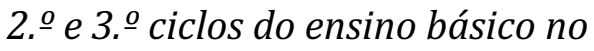
Minho (Tese de mestrado não publicada). Instituto Politécnico de Viana do Castelo, Viana do Castelo.

Baptista, I. (2014). Promoção da saúde em contexto escolar - modelos de intervenção (Dissertação de doutoramento não publicada). Universidade Técnica de Lisboa, Lisboa.

Baptista, I., \& Matos, M. G. (2011a). Educação sexual em meio escolar em portugal: avaliação da acção entre 2000 e 2005 (I Parte). Revista de psicologia da criança e do adolescente/Journal of child and adolescent psychology, 3, 77 -95.

Baptista, I., \& Matos, M. G. (2011b). Educação sexual em meio escolar: avaliação da acção entre 2005 e 2010 (II Parte). Revista de psicologia da criança e do adolescente/Journal of child and adolescente psychology, 4, 109 -124.

Becker, D., Edmundo, K., Nunes, N. R.., Bonnato, D., \& Souza, R. (2004). Empowerment e avaliação participativa em um programa de desenvolvimento local e promoção da saúde. Ciência \& Saúde Coletiva, 9, 655-667. doi:10.1590/S141381232004000300017

Bronfenbrenner, U. (1979). The ecology of human development: experiments by nature and design. Cambridge, MA: Harvard University Press.
Bronfenbrenner, U. (1988). Interacting systems in human development. Research paradigms: present and future. In U. Bronfenbrenner (Ed.), Making human beings human. Bioecological perspectives on human development (pp. 67-93). Thousand Oaks, CA: Sage Publications.

Bronfenbrenner U. (2005). Making human beings human. Bioecological perspectives on human development. Thousand Oaks, CA: Sage Publications.

Campos, B. P. (1991). Psychological development and personal and social education in the schools. In $\mathrm{H}$. Starkey (Ed.), Socialization of school children and their education for human values and democratic rights (pp.5376). Amsterdam: Swetz \& Zeitlinger.

Dias, S. (2013). Educação sexual nas escolas do concelho de Oeiras: Perceção de professores e alunos (Tese de mestrado não publicada). Universidade técnica de Lisboa, Lisboa

Eccles, J. S., \& Roeser, R. W. (2010). An ecological view of schools and development. In J. L. Meece \& J. S. Eccles (Eds.), Handbook of research on schools, schooling, and human development (pp. 6-21). New York: Routledge.

Eisenberg, M. E., Madsen, N., Oliphant, J. A., \& Resnick, M. (2012). Policies, principals and parents: multilevel challenges and supports in teaching sexuality education. Sex Education, 12, 317-29. doi: 10.1080/14681811.2011.615614 
European Network of Health Promoting Schools, WHO, \& European Commission (1997). First Conference of the European Network of Health Promoting Schools: the health promoting school - an investment in education, health and democracy: conference resolution, Thessaloniki-Halkidiki, Greece, 1-5 May 1997. Retirado de http://apps.who.int/iris/handle/10 665/107850\#sthash.dXAssZt2.dpuf

Fawcett, S. B., Paine-Andrews, A., Francisco, V. T., Schultz, J. A. Richter, K. P., Lewis, R. K., ... Lopez, C. M. (1995). Using empowerment theory in collaborative partnerships for community health and development. American Journal of Community Psychology, 23, 677-697. doi: 10.1007/BF02506987

Foster-Fishman, P. G., \& Keys, C. BS. (1997). The Person/Environment dynamics of employee empowerment: an organizational culture analysis. American Journal of Community Psychology, 25, 345-369.

Gómez-Zapiain, J., Sánchez, A., Quintana, P., \& Fernández, J. (2005). Programa de educación afectivo-sexual, uhin bare. Vitoria-Gasteiz: Universidad del País Vasco.

GTES (2005). Relatório preliminar. Lisboa: Ministério da Educação. Retirado de: http://www.netprof.pt/pdf/Relator io_EduSexual.pdf

GTES (2007). Relatório final. Lisboa: Ministério da Educação. Retirado de: http://www.dge.mec.pt/sites/defau lt/files/ficheiros/relatorio_final_gte s.pdf
Illback, R. J., Zins, J. E., Maher, C. A., \& Greenberg, R. (1990). An overview of principles and procedures of program planning and evaluation. In T. B. Gutkin \& C. R. Reynolds (Eds.). The handbook of school psychology, (pp. 799-820). New York: Wiley.

IPPF (2006). Sexuality education in Europe: a reference guide to policies and practices. Brussels: IPPF European Network.

IPPF (2010). Framework for comprehensive sexuality education. London: IPPF.

Ketting, E., \& Winkelmann, C. (2013). New approaches to sexuality education and underlying paradigms. Bundesgesundheitsblatt, Gesundheitsforschung, Gesundheitsschutz, 56, 250-55. doi: 10.1007/s00103-012-1599-8

Kirby, D. (2008). The impact of abstinence and comprehensive sex and STD/HIV education programs on adolescent sexual behavior. Sexuality Research \& Social, 5, 18-27. doi: 10.1525/srsp.2008.5.3.18

Leal, C. (2013). Educação sexual no contexto escolar português: $A$ implementação em duas direções regionais de educação (Tese de mestrado não publicada). Universidade do Porto, Porto.

Loeber, O., Reuter, S., Apter, D., van der Doef, S., Lazdane, G., \& Pinter, B. (2010). Aspects of sexuality education in Europe - definitions, differences and developments. The European Journal of Contraception \& Reproductive Health Care, 15, 16976. doi: 10.3109/13625181003797280

Macário, R. (2010). Educação sexual em contexto escolar: Outro ano zero?! (Tese de mestrado não publicada). Universidade do Algarve, Faro. 
Marques, A. M., Pereira, A., Silva, B., Vilar, D., \& Cadete, J. (1999). Orientações técnicas sobre a educação sexual em meio escolar. Lisboa: PES/APF/DGS.

Matos, M. G., Ferreira, M., Tomé, G., Camacho, I., Reis, M., Baptista, I., ...Diniz, J. A. (2011). Condições ambientais, pedagógicas e psicossociais nas escolas: Uma visão da gestão escolar e sua evolução em 4 anos. Revista de Psicologia da Criança e do Adolescente, 3, 111125.

Matos, M. G., Reis, M., Ramiro, L., Ribeiro, J. L., Leal, I., \& Equipa Aventura Social (2013). Relatório final avaliação do impacto da lei $n .{ }^{\circ}$ 60/2009, de 6 de agosto,

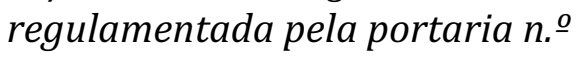
196-a/2010, de 9 de abril. Retirado de: www.dge.mec.pt/sites/default/files /.../relatorio_es_lei_dgs_spps_final.p df

McMillan, D. W., \& Chavis, D. M. (1986). Sense of community: a definition and theory. Journal of Community Psychology, 14, 6-24.

Menezes, I. (2010). Intervenção comunitária: uma perspetiva psicológica. Porto: Livpsic/Legis Editora.

Ministério da Educação (2009). Edital 2008/2009 - Resultados. Retirado de: http://www.dgidc.minedu.pt/educacaosaude/data/educac aosaude/promocaoeducacaosaude/ edital2008_2009.pdf

Ministério da Educação (2010). Edital 2009/2010 - Resultados. Retirado de: http://www.dgidc.minedu.pt/educacaosaude/data/educac aosaude/promocaoeducacaosaude/ edital2009_2010_avalfinal.pdf
Ministério da Educação (2011). Edital 2010/2011 - Resultados. Retirado de: http://www.dgidc.minedu.pt/educacaosaude/data/educac aosaude/promocaoeducacaosaude/ edital2010_2011_resultados.pdf

Ministério da Educação, Ministério da Saúde, \& APF (2000). Educação sexual em meio escolar: Linhas orientadoras. Lisboa: ME/MS.

Montero, M. (2004).Introduccion a la psicologia comunitária. Buenos Aires: Paidós.

Nelson, G., \& Prilleltensky, I. (2005). Community psychology: in pursuit of liberation and well-being. New York: Palgrave Macmillan

Oja, S. N., \& Smulyan, L. (1989). Collaborative action research: $a$ developmental approach. Sussex: The Falmer Press.

Orford, J. (1992).Community psychology. Theory and practice. Chichester: Wiley.

Parker, R., Wellings, K., \& Lazarus, J. V. (2009). Sexuality education in Europe: An overview of current policies. Sex Education, 9, 227-42. doi: 10.1080/14681810903059060

Ramiro, L. (2013). A educação sexual na mudança de conhecimentos, atitudes e comportamentos sexuais dos adolescentes (Dissertação de doutoramento não publicada). Universidade Técnica de Lisboa, Lisboa.

Ramiro, L., Reis, M., Matos, M. G., \& Diniz, J. A. (2011). Sex education among Portuguese adolescent students. Procedia - Social and Behavioral Sciences, 29, 493-502. doi: 10.1016/j.sbspro.2011.11.268

Ramiro, L., Reis, M., Matos, M. G., \& Diniz, J. A. (2013). Perceções de professores e pais/mães sobre educação para a saúde e educação sexual na família e nas escolas 
portuguesas. Saúde Reprodutiva, Sexualidade e Sociedade, 3, 37-45.

Ramiro, L., Reis, M., Matos, M. G., Diniz, J. A., Ehlinger, V., \& Godeau, E. (2014). Sexually transmitted infections prevention across educational stages: Comparing middle, high school and university students in Portugal. Creative Education, 5, 1405-17. doi: 10.4236/ce.2014.515159

Ramos, S. (2008). Ouvindo o Bairro: o sentido psicológico de comunidade num bairro social (Tese de mestrado não publicada). Universidade do Porto, Porto.

Rappaport, J. (2005). Community psychology is (thank God) more than science. American Journal of Community Psychology, 35, 231-238.

Reis, M. (2012). Promoção da saúde sexual em jovens universitários portugueses - Conhecimentos e atitudes face à contraceção e à prevenção das ISTs (Dissertação de doutoramento não publicada). Universidade Técnica de Lisboa, Lisboa.

Reis, M., Ramiro, L., Matos, M. G., \& Diniz, J. A. (2011). The effects of sex education in promoting sexual and reproductive health in Portuguese university students. Procedia Social and Behavioral Sciences, 29, 477-85. doi: 10.1016/j.sbspro.2011.11.266

Reis, M., Ramiro, L., Matos, M. G., \& Diniz, J. A. (2013). Educação sexual e estudantes do ensino superior- a importância da educação sexual e a influência da família, da escola e dos amigos na adoção de comportamentos sexuais saudáveis nos estudantes do ensino superior. Saúde Reprodutiva, Sexualidade e Sociedade, 3, 20-27.
Rocha, A. C. (2015). Educação sexual no contexto escolar português: caraterização, facilitadores e barreiras à sua implementação (Tese de doutoramento não publicada). Universidade do Porto, Porto.

Rocha, A. C., Leal, C., \& Duarte, C. (2015). School-based sexuality education in Portugal: Strengths and weaknesses. Sex education, early online. doi: 10.1080/14681811.2015.1087839

Rocha, A. C., \& Duarte, C. (2015a).

Sexuality education in a representative sample of Portuguese schools: examining the impact of legislation. The European Journal of Contraception \& Reproductive Health Care, 20, 47-56. doi:10.3109/13625187.2014.95199 6

Rocha, A. C., \& Duarte, C. (2015b). Factors facilitating implementation of school-based sexuality education in Portugal. The European Journal Contraception Reproductive Health Care, early online, 1-9. doi: 10.3109/13625187.2015.1057809

Sánchez, F. L. (2005). La educación sexual. Madrid: Biblioteca Nueva.

Sousa, A. P., Soares, I., \& Vilar, D. (2007). Lessons learnt from a secondary school sex education program in Portugal. Sex Education, 7, 35-45. doi: 10.1080/14681810601134835

Sousa, R. (2012). Um olhar sobre o contexto da educação sexual em Portugal. Saúde Reprodutiva, Sexualidade e Sociedade, 2, 4-23.

UNESCO (2009). International technical guidance on sexuality education: an evidence-informed approach for schools, teachers and health educators. Paris: UNESCO. 
UNESCO (2010). Levers of success: case studies of national sexuality education programmes. Paris: UNESCO.

Vaz, J. M., Vilar, D., \& Cardoso, S. (1996). Educação sexual na escola. Lisboa: Universidade Aberta.

Vilaça, T. (2013). Perspectiva evolutiva das políticas e práticas de educação sexual na comunidade escolar em Portugal. Doxa, 17, 245-293.

UIPES (2010). Promover a saúde na escola: da evidência à acção. Retirado de http://www.iuhpe.org/images/PUB LICATIONS/THEMATIC/HPS/Evide nce-Action_POR.pdf

WHO/BZgA (2010). Standards for sexuality education in Europe: A framework for policy makers, educational and health authorities and specialists. Cologne: WHO/BZgA.

WHO/BZgA (2013). Standards for sexuality education in Europe: Guidance for implementation. Cologne: WHO/BZgA.

Zimmerman, M. A. (1995). Psychological empowerment: issues and illustrations. American Journal of Community Psychology, 23, 581-599. 